\title{
Aggregated Color Descriptors for Land Use Classification
}

\author{
Vedran Jovanović and Vladimir Risojević
}

\begin{abstract}
In this paper we propose and evaluate aggregated color descriptors for land use classification in aerial images. First, global and local Bag-of-Colors (BoC) descriptors are evaluated for land use classification. Influence of different parameters on the performance and efficiency of classification were tested. A small modification in the process of computing of $\mathrm{BoC}$ is introduced, which improves the overall classification performance. We also present a new, very simple color descriptor, termed Vector of Locally Aggregated Colors (VLAC) which has as good classification results as the modified $\mathrm{BoC}$, but using linear support vector machines.
\end{abstract}

Keywords - BoC descriptor, VLAC descriptor, color descriptors, Land Use Classification

\section{INTRODUCTION}

$\mathrm{I}_{\mathrm{s}}^{\mathrm{s}}$ this paper we consider the problem of land use classification in high-resolution overhead imagery. The main challenge in this area consists of finding a powerful descriptor and classifier, which will distinguish different types of images, based on their content and assign them into different classes, and on the other hand, be invariant to all transformations and factors that can decrease the quality of image such as cropping, rotation, scaling, noise, etc.

There are a vast number of papers related to descriptor generation. Many of them are based on using descriptors extracted locally from image patches, in particular Scale Invariant Feature Transform (SIFT) descriptor and its modifications [1], [2]. These descriptors are often jointly used with Bag-of-Visual-Words (BoVW) framework, where, starting from a set of local descriptors, a fixed size vector for each image is computed.

Another way for combining local descriptors into a fixed size vector is Vector of Locally Aggregated

Paper received July 21, 2015; revised October 7, 2015; accepted October 13, 2015. Date of publication November 15, 2015. The associate editor coordinating the review of this manuscript and approving it for publication was Prof. Irini Reljin.

This paper is a revised and expanded version of the paper presented at the 22th Telecommunications Forum TELFOR 2014.

This research was supported in part by Norwegian Ministry of Foreign Affairs through HERD ICT NORBOTECH (NORwayBOsnia TECHnology Transfer) project for Programme in Higher Education, Research and Development project under contract 2011/1370, and in part by the Ministry of Science and Technology of the Republic of Srpska under contract 19/6-020/961-187/14.

V. Jovanović, University of Banja Luka. Faculty of Electrical Engineering, Patre 5, 71000 Banja Luka, Bosnia and Herzegovina (tel: +38751-221-874, e-mail: vedran.jovanovic@etfbl.net).

V. Risojević, University of Banja Luka, Faculty of Electrical Engineering, Patre 5, 71000 Banja Luka, Bosnia and Herzegovina (tel: +38751-221-847, e-mail: vlado@etfbl.net).
Descriptors (VLAD) framework, introduced by Jegou et al. [3], [4], which reduces the amount of data needed to obtain a final descriptor, without noticeably impacting its accuracy [5], [6]. VLAD descriptors show excellent performance and accuracy in systems for large-scale image search and retrieval [3] - [6].

Although very powerful, most of descriptors based on SIFT work only with gray-level images, and do not take into account color information.

Color is a very expressive visual feature, and as such, it could be a very helpful cue in systems for recognition and object detection, since different object types have different colors. One notable example is land cover/land use classification in remote sensing images, where different land cover types have different colors (forest and grass are green, water is blue, etc.).

One of the first color descriptors was described in [7], where a color histogram was introduced. Since then, different color descriptors have been presented, and some of them are also included in the MPEG-7 standard for the description of audio-visual content [8].

In [9] the Bag-of-Colors (BoC) descriptor has been introduced that uses an adaptive approach for color codebook computation, in contrast to fixed color partition, traditionally used with color descriptors. Then, this descriptor was tested in [10] for use in aerial image classification.

The main contributions of this paper are:

- BoC descriptor for land use classification is evaluated,

- Modification in the $\mathrm{BoC}$ computation process is proposed, which improves classification performance,

- New color descriptor, termed Vector of Locally Aggregated Colors (VLAC) is introduced,

- Influence of different parameters and normalization techniques on classification accuracy is tested.

The paper is organized as follows. In Section II we describe the ways of computing global and local BoC descriptors. A procedure for computing VLAC descriptor is introduced in Section III. In Section IV we describe the used image dataset, methodology of testing, and present the results obtained by testing both descriptors. In Section $\mathrm{V}$ we give concluding remarks and suggest future work.

\section{BOC DESCRIPTOR}

There are two different ways of BoC computation. If the descriptor is extracted from the whole image, a global $\mathrm{BoC}$ is obtained, while if the descriptors are computed for a set of image patches, we obtain local descriptors. 


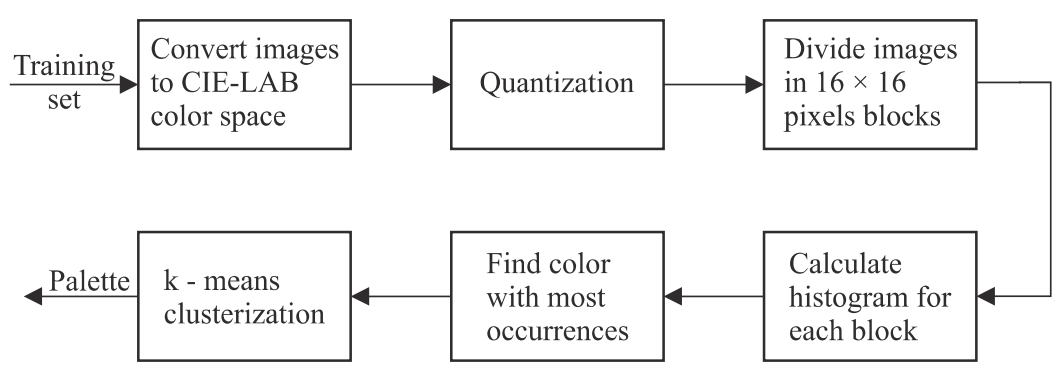

Fig. 1. Block scheme for palette computation process.

\section{A. Global BoC descriptor}

A global descriptor is generated from the whole image. The first step in the process of descriptor computation is generation of a color codebook (palette). The process of palette generation is shown in Fig. 1. First, each image from a training set is converted to CIE-LAB color space and each color component is uniformly quantized in $N$ bins. After this, each image is divided into blocks of the size of $16 \times 16$ pixels, and the color with most occurrences is extracted for each block. Then, all colors extracted in this way have been clustered using k-means algorithm producing a color codebook with $k_{c}$ colors. In this way we have learned a palette which is more adjusted to real-world images than fixed color space quantization. A palette learned with parameters $N=8$ and $\quad k_{c}=100$ is shown in Fig. 2. As a product of palette learning and unbalanced color statistic of aerial images, some colors such as green, gray and brown have bigger contributions to the palette than others, e.g. red or blue.

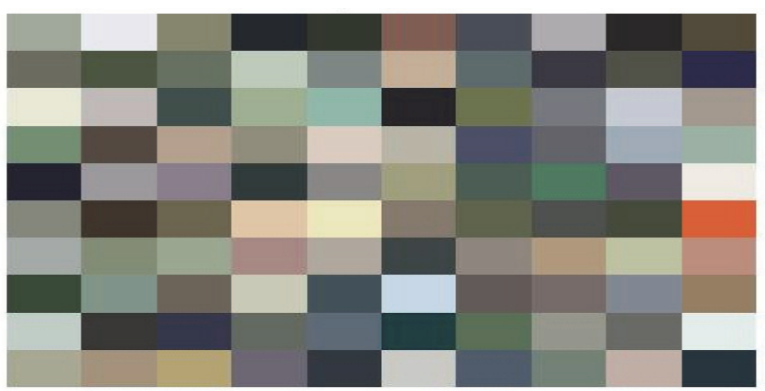

Fig. 2. Color palette obtained after learning on real-world aerial images $\left(N=8, k_{c}=100\right)$.

When the palette is obtained, the next step is BoC generation. Similarly to BoVW, we compute image representation as a histogram of codeword occurrences for each image with regard to the specified palette. This is done in a way that for each pixel from image we calculate its Euclidean distances to the colors in the palette, whereupon we increment the histogram bin corresponding to the color with the shortest distance. After this, each image is represented with a fixed-size vector of length $k_{c}$ a global BoC descriptor.

As the last step, different types of normalization techniques have been tested in order to achieve better results. Many of these techniques are also used jointly with SIFT-based descriptors. We have tested inverse-document frequency (IDF), power-law, L1 and L2 normalizations, and their combinations. The best results have been achieved using only L1 normalization, which is used in further work.

\section{B. Local BoC descriptor}

The process of computing image representation based on local BoC descriptor is summarized in Fig. 3. The first step is palette generation, which is performed in the same way as described before. This palette will be used later for the computation of patch descriptors. The second step is preparation of the input image for descriptor computation, where the image is converted into CIE-LAB color space and quantized by each color component regularly in $N$ bins. In this part we have also tested the influence of using a power-law transform before descriptor computation [2]. We have come to a conclusion that using the square root value of each color component instead of original color information decreases the performance of the final descriptor.

Similarly to a global BoC, the next step is the computation of $\mathrm{BoC}$ descriptor for each patch of the image, with regard to the palette. These patches can be obtained in many ways. They can be extracted by a region detector, regularly sampled, etc. In this paper, patches are regularly sampled.

Now, each image is represented with a bag of local BoC descriptors. In order to compute the codebook of local BoC descriptors, we apply k-means algorithm to descriptors obtained from images from a training set. The length of obtained codebook after clustering is $M$.

The last step of this process is image representation extraction. In this case image representation is actually a histogram of codeword occurrences with regard to the codebook of local BoC descriptors. For each image, which is represented with a bag of local $\mathrm{BoC}$ descriptors, we calculate Euclidean distances between these descriptors and codebook centroids and increment the bin in the final histogram, corresponding to the centroid with the shortest distance. At the end of this process each image is represented with a single vector of length $M$.

\section{VLAC DESCRIPTOR}

Similarly to BoC, we propose both global and local versions of the VLAC descriptor.

\section{A. Global VLAC descriptor}

In order to extract a global VLAC descriptor, we need to generate the color palette. After palette generation, instead of histogram computation as we did in $\mathrm{BoC}$ framework, we use VLAD approach in order to produce the final descriptor. Standard VLAD representation is 


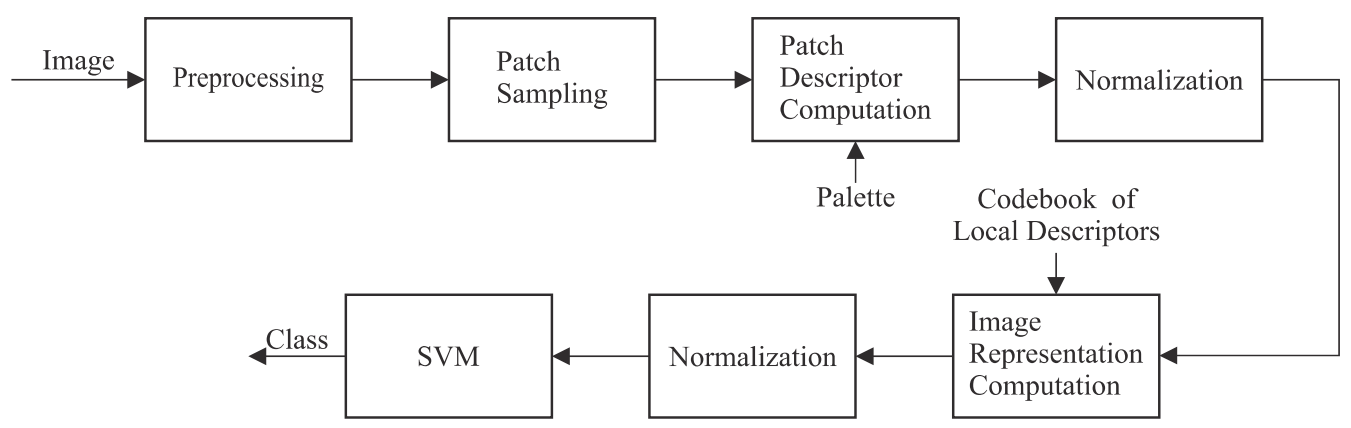

Fig. 3. Block scheme for computing image representation based on local BoC and VLAC image descriptors.

formed as a sum of all residual vectors, which represent differences between the local descriptor and the centroid it is assigned to [3]. In our case, for each of $k_{c}$ colors in palette $\left\{\boldsymbol{\mu}_{1}, \boldsymbol{\mu}_{2}, \ldots \boldsymbol{\mu}_{\mathrm{k}_{\mathrm{c}}}\right\}$, the differences $\mathbf{x}_{t}-\boldsymbol{\mu}_{i}$ of the vectors $\mathbf{x}_{t}$ assigned to $\boldsymbol{\mu}_{i}$ are accumulated:

$$
\mathbf{v}_{i}=\sum_{\mathbf{x}_{t}: N N\left(\mathbf{x}_{t}\right)=i} \mathbf{x}_{t}-\boldsymbol{\mu}_{i}
$$

where $\mathbf{x}_{t}$ represents the color of the $t$-th pixel, and $\mathrm{NN}\left(\mathbf{x}_{t}\right)$ represents its nearest cluster centroid. After this, the global VLAC descriptor is obtained by concatenating the residual vectors $\mathbf{v}_{i}$ into a single vector. Dimensionality of $\mathbf{x}_{t}$ is 3 ( $L$, $a, b$ color component), and it will define the dimension of each vector $\mathbf{v}_{i}$. Since there are $k_{c}$ centroids (one for each color in the palette), the length of the final vector obtained after concatenation is $k_{c} \times 3$.

After this, we have tested different normalization methods which are commonly used with VLAD framework. L2, power-law transform and normalized components [5] have been tested and the best results have been achieved using only L2 normalization.

\section{B. Local VLAC descriptor}

A block diagram which describes the computation of local VLAC descriptor is shown in Fig. 3. This process starts with color palette generation in the same way we have already explained. Preprocessing methods and patch extraction for this method are the same as described in Section II.B. The only difference is that, in this case, contrary to BoC, the use of square rooted values of colors does not influence the classification results.

In the patch descriptor calculation block, instead of the calculation of local histograms for each patch, as we did in BoC framework, in this method, we calculate VLAC representations of patches, given the color palette in the same way as described in (1). Now, each patch is represented with a VLAC descriptor of length $k_{c} \times 3$. Each image is represented with a bag of local VLAC descriptors, whose number is equal to the number of patches extracted from the image.

Applying the k-means algorithm to all local VLAC descriptors computed for training set images, will result in a codebook of local VLAC descriptors of length $M$. In order to produce a final fixed-size image descriptor, in image representation calculation block, we extract a VLAD descriptor for each image using its bag of local VLAC descriptors and codebook of local VLAC descriptors obtained using the k-means algorithm. The obtained vector is image representation based on local VLAC descriptors and its length is $K=3 \times k_{c} \times M$.

\section{EXPERIMENTS}

\section{A. Dataset and methodology}

In order to test effectiveness of the proposed descriptors we have chosen UC Merced Land Use (UCMLU) dataset. UCMLU is a set of aerial images, manually extracted from USGS National Urban Area Imagery collection, and divided into 21 classes, depending on different land covers and objects shown in them. Each class consists of 100 images, with a size of $256 \times 256$ pixels, and spatial resolution of $30 \mathrm{~cm}$ per pixel. Name and one sample of each class are depicted in Fig. 4.

For classification with both descriptors, we have chosen Support Vector Machine (SVM) classifier. We have tested linear SVM without and with nonlinear $\chi^{2}$ descriptor mapping [11]. The drawbacks of nonlinear mapping are higher memory usage and slower training, since it increases the dimensionality of final descriptor. The process of testing starts with dividing images randomly into training and test sets, where 80 images from each class were assigned to training and 20 to a test set. After this, we extract the color palette (for both BoC and VLAC descriptors), codebook of local $\mathrm{BoC}$ descriptor, and codebook of local VLAC descriptors only using training images, whereupon we calculate the final descriptor as a representation for each image.

Then, normalization is applied to descriptors $\overline{\mathbf{x}}=\mathbf{x} /\|\mathbf{x}\|_{p}$, where $p=1$ for L1, and $p=2$ for L2 normalization. We found out that L1 normalization is better in combination with $\mathrm{BoC}$ descriptor, while L2 normalization is preferred with VLAC descriptor.

As an accuracy measure, we have calculated the ratio of correctly classified test images and their total number. Ratio is computed for ten different training/test set splits, whereupon the classification accuracy for each set of parameters is presented with a mean value and standard deviation of accuracies for different dataset splits.

\section{B. BoC descriptor}

A summary of preliminary results obtained using BoC descriptor presented in [10] is shown in Table 1. As we can see, classification accuracy of standard BoC descriptor $(\mathrm{S})$, is very dependent on both the number of quantization 


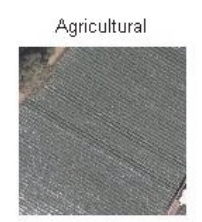

Forest

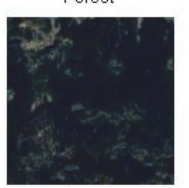

Overpass

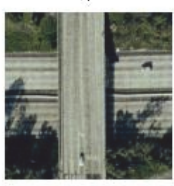

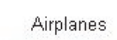

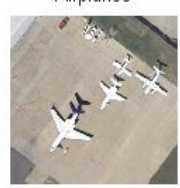

Freeway

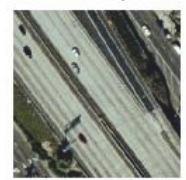

Parking Lot

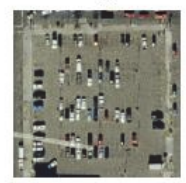

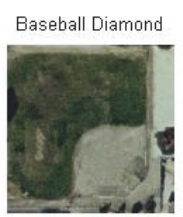

Golf Course

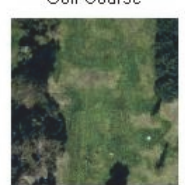

River

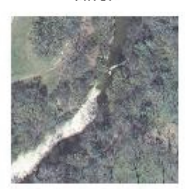

Beach

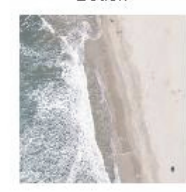

Harbor

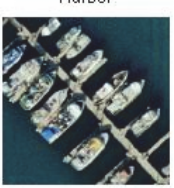

Runway

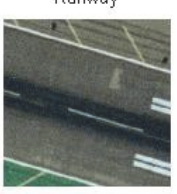

Buildings

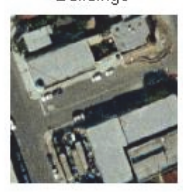

Intersection

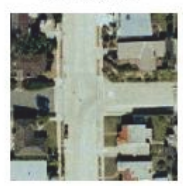

Sparse Residential

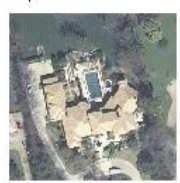

Chaparral

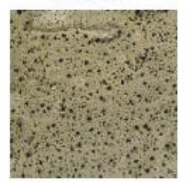

Medium Density Residential

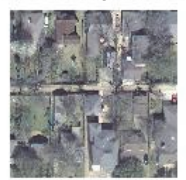

Storage Tanks

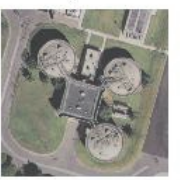

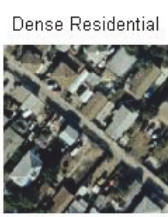

Mobile Home Park

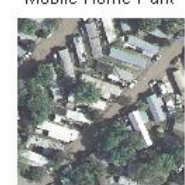

Tennis Courts

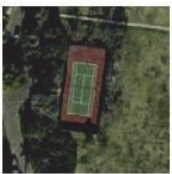

Fig. 4. UC Merced Land Use Dataset classes with an example.

levels, $N$, and the size of palette, $k_{c}$. The reason for this is the following. With increasing $k_{c}$ we increase the number of different colors that could be found in a palette, and also we increase diversity among the shades of colors which is very useful in order to distinguish images which are globally colored the same. For example, to distinguish grass from forest, information only about green color is not enough. However, if we have available more shades of green, we will probably be able to distinguish these two classes since the grass is much lighter than forest on images. On the other hand, increasing the number of quantization levels decreases the loss of color information on image, which also makes influence on different color shades in a palette. Besides influencing the classification accuracy, these parameters also influence classification time, which increases with increasing of $N$ or $k_{c}$. For example, if $k_{c}$ increases, the number of centroids in the process of k-means clustering increases, and it will take more time to find the nearest clusters. Also, a greater $k_{c}$ means higher dimensionality of final image descriptor, which will also increase the time of classification.

In order to eliminate the dependence of classification accuracy on parameter $N$, we introduce a small modification into the process of palette generation. Instead of using the whole color space range of an image in the process of quantization (in MATLAB for CIE-LAB color space this range is $[0,100]$ for $L$ component and $[-128,127]$ for $a$ and $b$ color components), we compute the color range for the quantization of each image dynamically, using the extreme intensity values for each image component separately (for image $i$, quantization range is calculated as $\left[\min \left(L_{i}\right), \max \left(L_{i}\right)\right]$ for $L,\left[\min \left(a_{i}\right)\right.$, $\left.\max \left(a_{i}\right)\right]$ for $a$, and $\left[\min \left(b_{i}\right), \max \left(b_{i}\right)\right]$ for $b$ color component, where $L_{i}, a_{i}, b_{i}$ are color components of the $i$ th image in CIE-LAB color space). As we can see in Table 1 the classification accuracy of this modified method (M) is still very dependent on parameter $k_{c}$, while parameter $N$ does not have almost any influence on accuracy. The reason for this lies in the fact that each image brings its own range of colors, which differs from image to image, and therefore, a large number of different colors contribute to the process of clustering. For a standard $\mathrm{BoC}$, the number of different colors in the process of clustering was always lower or equal to $N^{3}$, and from the results it is visible that increasing the length of the palette over $N^{3}$ does not improve the accuracy of classification. The advantage of our modified method is not only in improving the accuracy, but also in improving the performances of the whole system. In the palette generation process one step is finding a color with most occurrences in the histogram with $N^{3}$ elements (colors). If $N$ increases more memory is needed, and also it takes more time to search the histogram. In Table 1. we can see that classification accuracy over $86 \%$ for the modified method is achieved with $N=4$, while for a standard BoC, $N$ should be greater than 64 in order to achieve the same accuracy.

Results obtained for a local BoC descriptor are shown in Fig. 5. Some of these results were also presented in [10]. According to the conclusions we made for a global BoC descriptor, we chose the modified method for palette generation, in order to eliminate the dependency of results on parameter $N$. The number of quantization levels per color component was $N=8$. Patch was defined as a block of $16 \times 16$ pixels, which was moved over the image with a fixed step of 4 pixels.

From these results we can see that, similarly to a global $\mathrm{BoC}$, the length of final descriptor $M$ has a huge influence on classification results, and for better results we should a larger $M$. But, on the other hand, contrary to a global BoC, the maximum of classification accuracy, in this case, is obtained with a relatively small number of colors in the palette. We can see that the maximum of each curve is attained approximately for $k_{c}=50$. This is a consequence of patch size. In fact, the size of a patch is much smaller than the size of an image, and choosing a large value for $k_{c}$ will result in sparse local histograms in the patch descriptor computation part. Clustering of sparse vectors then results in large quantization errors, which reduce the classification accuracy. This influence is best visible in Fig. 5. for $k_{c}>100$, where the accuracy of classification drastically decreases, when $k_{c}$ increases. 
TABLE 1: ClassificATION ACCURACIES (\%) OBTAINED USING STANDARD GLOBAL BoC (S) AND MODIFIED GLOBAL BOC (M) DESCRIPTOR.

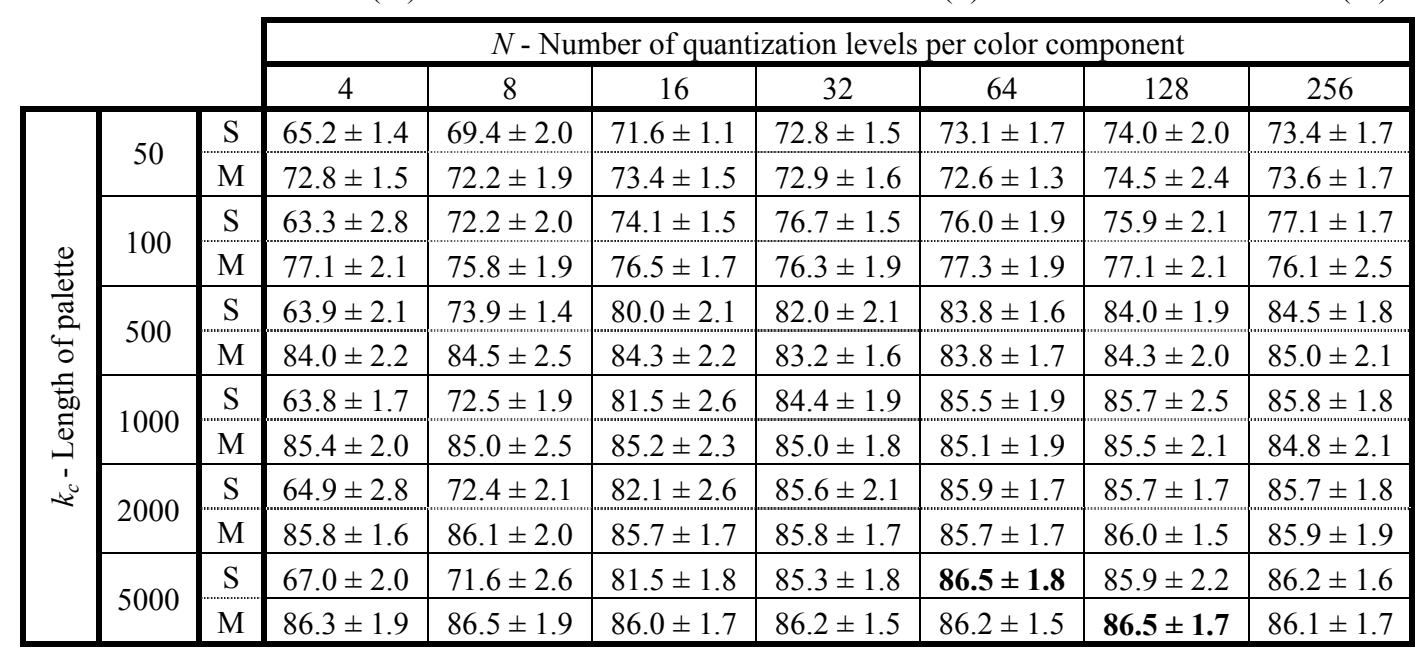

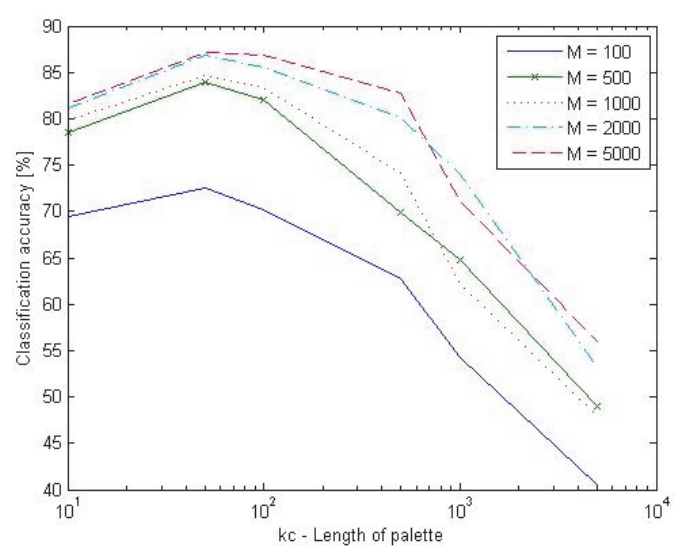

Fig. 5. Classification accuracies (\%) obtained using local BoC descriptor.

\section{VLAC descriptor}

For testing yhe VLAC descriptor we used a similar procedure as we did for the $\mathrm{BoC}$ descriptor in order to compare the results obtained using both methods. As described before, difference in the generation process between global BoC and global VLAC descriptors, is in the way in which a final descriptor is generated from the palette of colors. In BoC framework we calculated the histogram of colors with regard to the palette, resulting in the final descriptor of the same length as the palette, while in VLAC framework we used VLAD approach, which produces a final descriptor with three times larger size than the palette. In order to compare the results obtained using different frameworks, the palette used for global VLAC descriptor computation has three times less colors, compared to the palette used for global BoC descriptor computation, so the lengths of the final descriptors are the same. Comparative results are shown in Fig. 6. We can see that, for this dataset, the global BoC descriptor result in a better performance than VLAC. The reason for this lies in the fact that all images in a dataset are colorful, and many classes differ from others mostly in dominant colors, so a color histogram gives much more information about a picture than similarity to some color (which is the case in VLAC). In this case, we are not really interested in finding the shades of color, because if we want, for example, to distinguish trees from roads, it is enough to have information about the contribution of green and gray colors in BoC framework.

From Fig. 6. it can also be seen that if we only L2 normalize the global VLAC descriptor, better results are obtained using a nonlinear SVM classifier. However, when we apply a square root transform before L2 normalization, a linear SVM shows a better performance. One of the greatest advantages of using the VLAC descriptor is that it gives as good results as $\mathrm{BoC}$, but using a linear SVM, which takes less time to train compared to a nonlinear SVM, which is visible in Table 2. This will be even more significant when the local VLAC descriptor is considered.

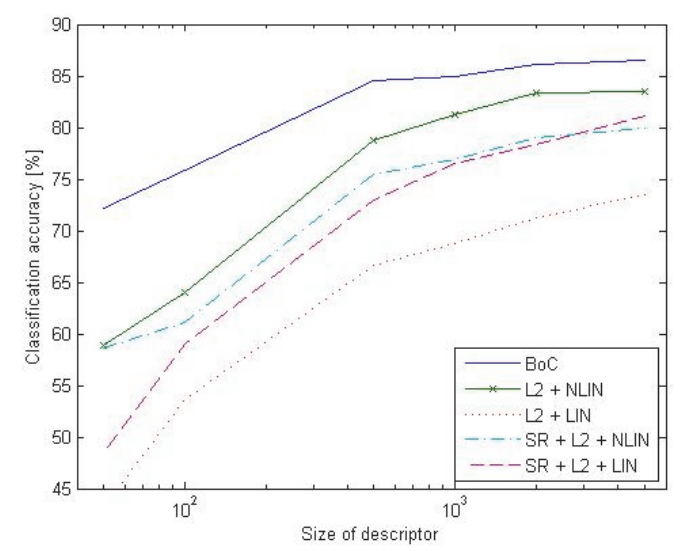

Fig. 6. Comparative overview of classification accuracies

(\%) obtained using global BoC and global VLAC

descriptor with different normalization techniques.

For testing the local VLAC descriptor the two main parameters, that were tested, were the size of palette $k_{c}$ and the size of codebook of locally aggregated colors $M$. The value of parameter $M$ was varied between 10 and 100 , while the value of parameter $k_{c}$ was calculated in such a way that the length of the final descriptor $K$ is closest to 5000. Obtained results are shown in Fig. 7. We used a linear SVM for classification, and according to the conclusions we made for a global VLAC descriptor, we used a square root transform and L2 normalization before a SVM classifier. 
Descriptor computation and classification is implemented in MATLAB 2013a, and tested on a computer with Intel Core i5 - 4300M CPU, 2.6 GHz, 4 cores, 8 GB RAM, Windows 7 - 64-bit.

The best results obtained using all four approaches are shown in Table 3. We can see that the best results are again obtained using a nonlinear SVM, but the results obtained using local VLAC descriptors and linear SVM are within one standard deviation. Moreover, they are similar to the best results obtained using a BoC descriptor with a nonlinear kernel. This can be seen in Fig. 8 and Fig. 9, where the confusion matrices for $\mathrm{BoC}$ and VLAC descriptors are depicted, respectively.

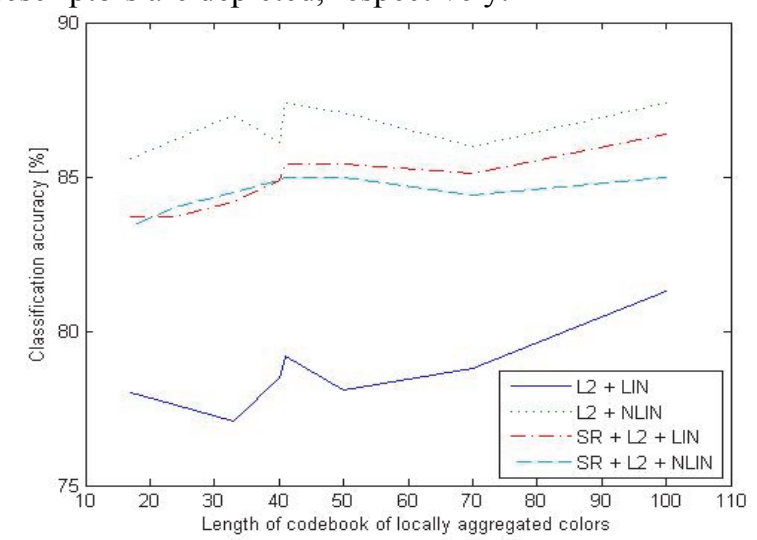

Fig. 7. Classification accuracies (\%) obtained using local VLAC descriptor with different normalization techniques.

TABLE 2: COMPUTATIONAL TIMES.

\begin{tabular}{|l|l|l|}
\hline \multirow{2}{*}{ Palette } & $N=8, k_{c}=5000$ & $81 \mathrm{~s}$ \\
\cline { 2 - 3 } & $N=8, k_{c}=50$ & $27 \mathrm{~s}$ \\
\hline Global BoC & \multicolumn{2}{|c|}{$0.3 \mathrm{~s}$} \\
\hline Local BoC & \multicolumn{2}{c|}{$1.3 \mathrm{~s}$} \\
\hline Global VLAC & \multicolumn{2}{c|}{$2.0 \mathrm{~s}$} \\
\hline Local VLAC & \multicolumn{2}{|c|}{$2.1 \mathrm{~s}$} \\
\hline \multirow{2}{*}{ Linear SVM } & Training & $34 \mathrm{~s}$ \\
\cline { 2 - 3 } & Classification & $0.2 \mathrm{~s}$ \\
\hline \multirow{2}{*}{ Nonlinear SVM } & Training & $79 \mathrm{~s}$ \\
\cline { 2 - 3 } & Classification & $0.6 \mathrm{~s}$ \\
\hline
\end{tabular}

TABle 3: ClASSIFICATION ACCURACIES (\%) OBTAINED WITH BOC AND VLAC DESCRIPTORS.

\begin{tabular}{|l|l|l|l|}
\hline \multirow{4}{*}{ BoC } & \multirow{2}{*}{ Global } & Linear & $62.8 \pm 2.4$ \\
\cline { 3 - 4 } & & Nonlinear & $86.5 \pm 1.7$ \\
\cline { 2 - 4 } & \multirow{2}{*}{ Local } & Linear & $78.1 \pm 2.6$ \\
\cline { 3 - 4 } & & Nonlinear & $87.1 \pm 1.0$ \\
\hline \multirow{3}{*}{ VLAC } & \multirow{2}{*}{ Global } & Linear & $81.1 \pm 2.1$ \\
\cline { 3 - 4 } & & Nonlinear & $83.5 \pm 2.0$ \\
\cline { 2 - 4 } & \multirow{2}{*}{ Local } & Linear & $86.4 \pm 1.9$ \\
\cline { 3 - 4 } & & Nonlinear & $\mathbf{8 7 . 6} \pm \mathbf{1 . 8}$ \\
\hline
\end{tabular}

\section{CONCLUSION}

In this paper we propose two very simple color descriptors for use in image classification. We find out that descriptors, obtained using only color information, can be a very powerful tool for achieving high classification accuracies. Results show that BoC and VLAC are very effective descriptors, and best of all is that they are very easy to calculate. Since the generation process of these descriptors takes into account only color information, in order to increase performance and accuracy, they could be combined with other descriptors such as SIFT, texture descriptors, etc.

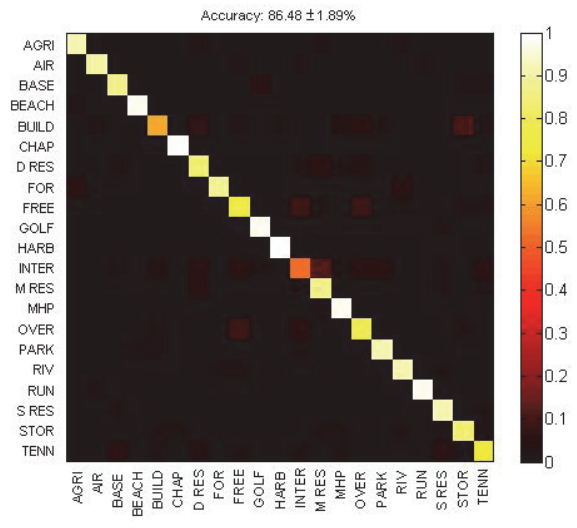

Fig. 8. Confusion matrix for global $\mathrm{BoC}$ descriptor with $M=5000$ and nonlinear SVM.

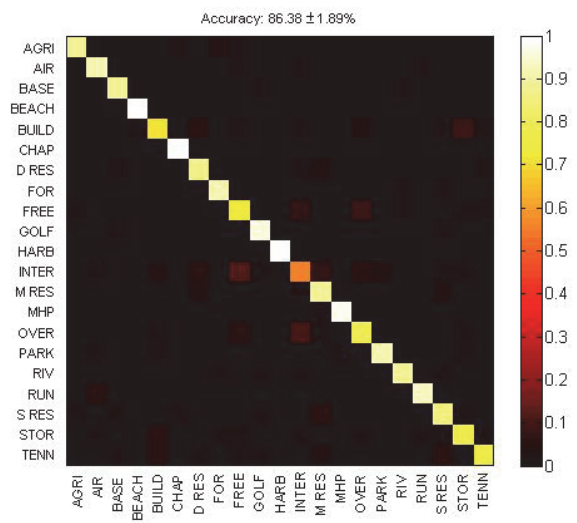

Fig. 9. Confusion matrix for VLAC descriptor with $K=5100$ and linear SVM.

\section{REFERENCES}

[1] Y. Yang and S. Newsam, "Bag-of-visual-words and spatial extensions for land-use classification", in ACM SIGSPATIAL GIS, 2010.

[2] R. Arandjelović, A. Zisserman, "Tree things everyone should know to improve object retrieval", Proc. IEEE Conf. Comput. Vis. Pattern Recognit., pp. 2911-2918, 2012.

[3] H. Jegou, M. Douze, C. Schmid, P. Perez, "Aggregating local descriptors into a compact image representation", Proc. IEEE Conf. Comput. Vis. Pattern Recognit., pp. 3304-3311, 2010.

[4] H. Jegou, F. Perronnin, M. Douze, J. Sanchez, P. Perez, C. Schmid, "Aggregating local image descriptors into a compact codes", IEEE Trans. Pattern Anal. Mach. Intell., vol.34, no.9, pp. 1704 - 1716, 2012.

[5] R. Arandjelović, A. Zisserman, "All about VLAD", Proc. IEEE Conf. Comput. Vis. Pattern Recognit., pp. 1578-1585, 2013.

[6] J. Delhumeau, P.H Gosselin, H. Jegou, P. Perez, "Revisiting the VLAD image representation", in Proc. ACM International Conference on Multimedia, pp. 653-656, 2013.

[7] M. J. Swain and D. H. Ballard, "Color indexing", Int. J. Comput. Vis., vol. 7, no. 1, pp.11-32, 1991.

[8] Manjunath, Bangalore S., et al. "Color and texture descriptors" IEEE Trans. Circuits Syst. Video Technol.,vol.11, no.6, pp. 703715,2001

[9] C. Wengert, M. Douze, H. Jegou, "Bag-of-colors for improved image search" Proc. ACM International Conference on Multimedia, pp. 1437 - 1440, 2011.

[10] V. Jovanović, V. Risojević, "Evaluation of Bag of Colors Descriptor for Land Use Classification", In Proc. of TELFOR 2014, pp.889 - 892.

[11] A. Vedaldi and A. Zisserman, "Efficient additive kernels via explicit feature maps", IEEE Trans. Pattern Anal. Mach. Intell., vol.34, no.3, pp. $480-492,2012$. 